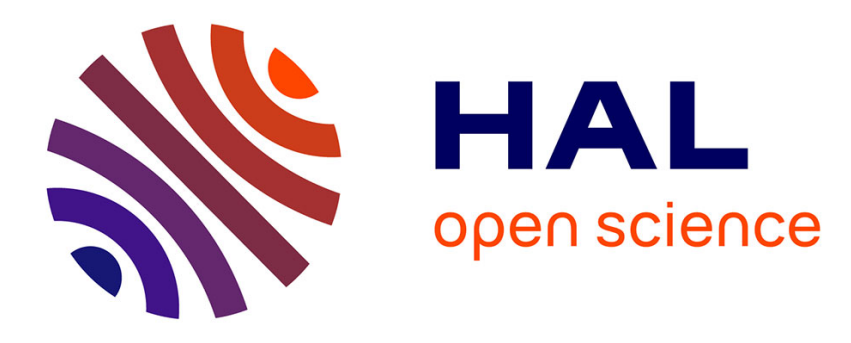

\title{
Modèles de commande pour asservissements électro-hydrauliques de position
}

\author{
M. Bertrand, J. Poulain
}

\section{To cite this version:}

M. Bertrand, J. Poulain. Modèles de commande pour asservissements électro-hydrauliques de position. Journal de Physique III, 1992, 2 (10), pp.1879-1889. 10.1051/jp3:1992219 jpa-00248852

\section{HAL Id: jpa-00248852 https://hal.science/jpa-00248852}

Submitted on 1 Jan 1992

HAL is a multi-disciplinary open access archive for the deposit and dissemination of scientific research documents, whether they are published or not. The documents may come from teaching and research institutions in France or abroad, or from public or private research centers.
L'archive ouverte pluridisciplinaire HAL, est destinée au dépôt et à la diffusion de documents scientifiques de niveau recherche, publiés ou non, émanant des établissements d'enseignement et de recherche français ou étrangers, des laboratoires publics ou privés. 
Classification

Physics Abstracts

$06.70 \mathrm{~T}-06.50 \mathrm{M}-47.60-47.90$

\title{
Modèles de commande pour asservissements électro- hydrauliques de position
}

\author{
M. Bertrand et J. Poulain \\ E.N.S.A.M., 8 Bd Louis XIV, 59000 Lille, France \\ (Reçu le 26 mars 1992, accepté le 16 juin 1992)
}

\begin{abstract}
Résumé. - L'article expose d'abord les principes de la modélisation à base physique des asservissements électro-hydrauliques. Il montre que les modèles obtenus sont complexes et mal adaptés aux moyens de commande industriels. Mais l'identification des réponses fournies par ces modèles, si elle est menée avec quelques précautions, fournit de bons modèles de commande. On peut alors élaborer plus sûrement des lois de commande permettant d'atteindre de hautes performances.
\end{abstract}

\begin{abstract}
The present paper expounds at first the principles of physical modelling of electrohydraulic servomechanisms. It shows that the obtained models are too complex and do not fit on industrial control means. But identification of the responses issued from these models provides, if achieved with some precautions, convenient control models. Control laws can then be more safely elaborated in order to reach high performances.
\end{abstract}

Les systèmes hydrauliques présentent beaucoup d'intérêt parce que leur rapport énergie transmise/masse est grand et que leur temps de réponse très bref permet de les utiliser même dans des applications où les puissances mises en jeu sont faibles (secteur biomédical par exemple). Mais, outre des difficultés techniques - utilisation d'huile que l'on cherche sans grand succès jusqu'ici à remplacer par des fluides «propres»-, la mise en place d'asservissements électro-hydrauliques se heurte au problème de la modélisation. La détermination d'algorithmes de commande simples et rapides à exécuter suppose un modèle mathématique lui-même simple, de préférence linéaire, minimisant l'étude nécessaire et autorisant l'emploi de matériel classique. Or l'analyse physique des systèmes hydrauliques fait apparaître des équations non linéaires dont, de plus, les coefficients ne sont parfois connus qu'avec une forte incertitude.

Nous allons nous efforcer d'apporter ici des solutions pour réduire cet inconvénient dans le cas d'asservissements à exigences élevées comportant une servovalve ou un servodistributeur. 


\section{La modélisation d'un système électro-hydraulique.}

Un système hydraulique-type comporte :

- une centrale hydraulique, source d'énergie, et ses auxiliaires (amortisseur, clapet de surpression, etc.) ;

- une servovalve et son dispositif de commande, généralement électrique, parfois pneumatique ;

- un actionneur (vérin ou moteur rotatif);

- une charge dont les caractéristiques peuvent parfois varier.

Les équations décrivant le fonctionnement d'un tel système traduisent la conservation des forces, celle des débits, le théorème de Bernoulli, les lois fondamentales de la thermodynamique.

Résumons ceci dans le cas d'une masse $M$ entraînée linéairement par un vérin commandé par une servovalve débit à 2 étages, le fluide sous pression provenant d'une centrale dont l'élément essentiel est une pompe à pistons axiaux (Fig. 1); ce cas est représentatif de nombreux problèmes, les variantes technologiques n'amenant pas de changements fondamentaux dans les équations représentatives du fonctionnement, et partant dans leur exploitation pour la commande. L'ordre suivi ira de la production d'énergie à sa mise en œuvre finale pour le déplacement avec positionnement de la charge.

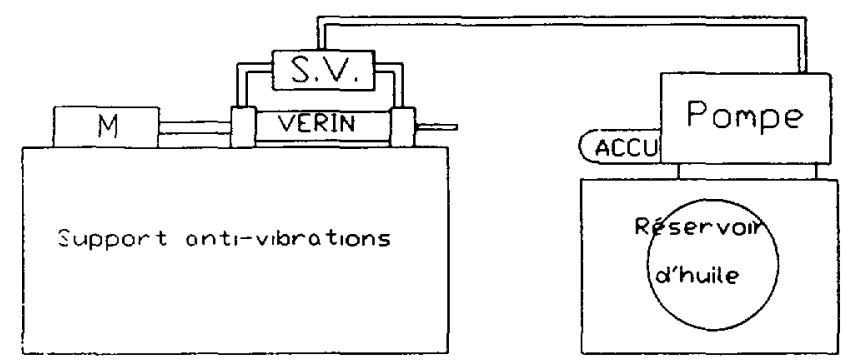

Fig. 1. - Schéma de principe de l'asservissement.

[Principle of the servomechanism.]

1.1 L'alimentation. - Elle a pour but de fournir un débit, la pression n'existant que par suite d'obstacles mis à ce débit. Le clapet de surpression joue à la fois le rôle d'obstacle et celui d'élément de régulation. On peut en dire autant de l'accumulateur à azote.

La pompe.

Pour une pompe à débit fixe, on a les relations suivantes :

$$
\frac{V_{\mathrm{r}}}{B} \frac{\mathrm{d} P_{\mathrm{r}}}{\mathrm{d} t}=-Q_{\mathrm{P}}+q_{\mathrm{f}}+\text { cyl } \frac{\mathrm{d} \theta}{\mathrm{d} t} \quad \text { côté refoulement },
$$

avec

$V_{\mathrm{r}}$ volume d'huile sous pression

$B$ module de compressibilité de l'huile

$P_{\mathrm{r}}$ pression côté refoulement

$Q_{\mathrm{P}}$ débit de la pompe 
$q_{\mathrm{f}}$ débit de fuite

cyl cylindrée de la pompe

$\theta$ position de l'arbre moteur.

Une équation de même type peut s'écrire côté aspiration, tandis que

$$
J \frac{\mathrm{d} \theta}{\mathrm{d} t}=r \operatorname{cyl}\left(P_{\mathrm{r}}-P_{\text {asp }}\right)-C_{\mathrm{R}}
$$

avec

$J \quad$ inertie de la pompe

$r$ rendement de la pompe

$C_{\mathrm{R}}$ couple résistant (frottement, couple statique).

L'accumulateur et le clapet de surpression.

Nous n'écrirons pas les équations pour ne pas alourdir la présentation; notons seulement que le clapet de surpression, en cas d'ouverture, introduit des termes dynamiques, et que l'accumulateur voit son rôle décrit par des équations de la thermodynamique fournissant la pression, le volume et la température du gaz, équations non linéaires même dans l'hypothèse où l'azote est considéré comme un gaz parfait, hypothèse insuffisante à des pressions de l'ordre de 200 bars maintenant couramment atteintes.

Simplification du rôle de l'alimentation.

Une interprétation simple du rôle de l'alimentation consiste à la définir comme un fournisseur de débit à pression constante.

Le rôle de l'accumulateur varie suivant qu'il constitue seulement un auxiliaire de l'alimentation - c'est le cas dans cette interprétation - ou qu'il est utilisé comme source transitoire d'énergie pour l'actionneur.

1.2 LA SERVOVALVE. - En négligeant divers phénomènes mineurs à caractère non linéaire :

- le «collage ", qu'on s'efforce d'annuler par envoi d'un courant sinusoïdal de faible amplitude mais de fréquence élevée (dither);

- les effets dynamiques des jets dans le système buse-palette, si la servovalve en comporte un ;

- les pertes de charge au passage dans les orifices internes;

- l'hystérésis du cycle magnétique,

ainsi que la dynamique de la partie électrique, la relation liant la sortie (débit $q_{1}$ vers le vérin) à l'entrée (courant $i$ fourni par l'électronique associée, elle-même pilotée par un régulateur), peut se mettre sous la forme

$$
q_{1}+2 \xi \tau_{n} \frac{\mathrm{d} q_{1}}{\mathrm{~d} t}+\tau_{n}^{2} \frac{\mathrm{d}^{2} q_{1}}{\mathrm{~d} t^{2}}=K_{\mathrm{s}} i
$$

équation différentielle linéaire du $2^{\mathrm{e}}$ ordre présentée de façon à faire apparaître une constante de temps $\tau_{n}$ et un coefficient d'amortissement $\xi$ généralement $<1$.

1.3 LE VÉRIN ET LA CHARGE. - En se limitant au cas le plus simple (vérin symétrique), schématisé sur la figure 2, le déplacement $x$ de la charge s'obtient par

$$
M \frac{\mathrm{d}^{2} x}{\mathrm{~d} t^{2}}=S\left(P_{1}-P_{2}\right)-f \frac{\mathrm{d} x}{\mathrm{~d} t}-F_{\mathrm{R}} \quad \text { (bilan des forces) }
$$




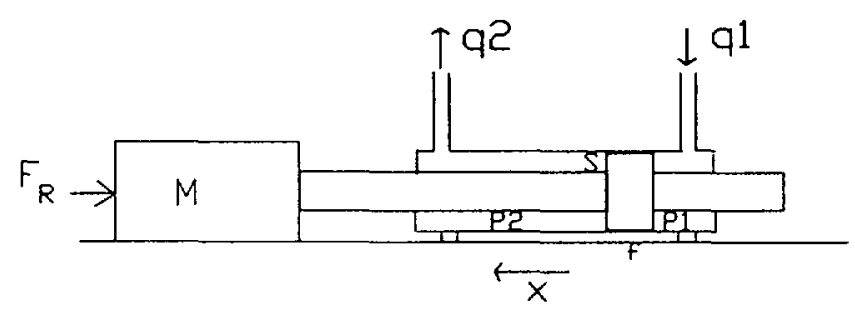

Fig. 2. - Le vérin et sa charge.

[The cylinder and its trained load.]

avec

- $S$ section utile du piston;

$-P_{1}$ pression côté arrivée du fluide ;

$-P_{2}$ pression côté retour du fluide;

$-f$ coefficient de frottement visqueux piston/chemise ;

$-F_{\mathrm{R}}$ force antagoniste.

Cette relation néglige les frottements secs et le terme proportionnel au carré de la vitesse dans l'expression du frottement visqueux. Par ailleurs,

$$
q_{1}=S \frac{\mathrm{d} x}{\mathrm{~d} t}+\frac{V+S x}{B} \frac{\mathrm{d} P_{1}}{\mathrm{~d} t}
$$

$V$ étant le $1 / 2$ volume d'huile et $P_{1}$ la pression en sortie de la servovalve si on néglige les pertes de charge dans les raccords.

Là encore, le système d'équations est non linéaire puisqu'apparaît un terme $x \mathrm{~d} P{ }_{1} / \mathrm{d} t$.

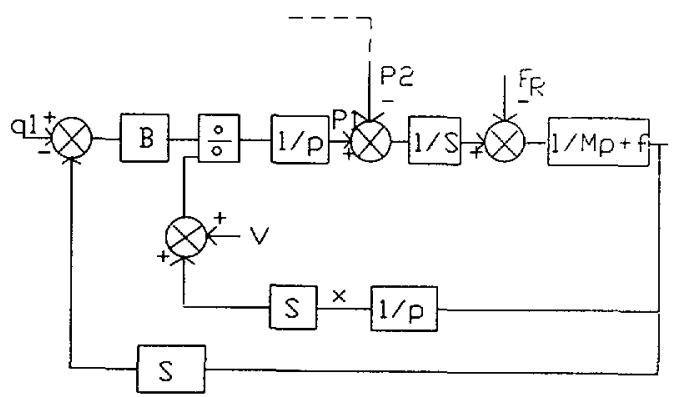

Fig. 3. - Schéma fonctionnel.

[Functional diagram.]

A noter que $M$ peut varier d'une application à une autre, voire au cours d'une même application.

1.4 Limites DE LA MOdÉlisation POUR LA COMMANDE. - Les équations précédentes, de par leurs imbrications, leurs non-linéarités qui ne sont pas toutes « séparables ", vont entraîner des lois de commande complexes ; outre le fait que ces algorithmes vont exiger une étude spécifique, la résolution de systèmes non linéaires, menée de manière itérative, n'est 
toujours pas réalisable, malgré les progrès des processeurs numériques, pour des systèmes à réponse rapide, où acquisitions et calculs doivent se dérouler en quelques millisecondes.

Les méthodes habituelles de commande résultent donc d'une simplification et d'une linéarisation des équations constituant le modèle physique. Cette procédure a priori, en présence de données incertaines, est contestable, même si de nombreuses méthodes se servent de linéarisations, à différents niveaux et plus ou moins complètement. L'identification va apporter des bases plus solides à la linéarisation, et permettre de l'appliquer quantitativement, en évaluant la précision des modèles retenus, et donc en limitant l'impact des simplifications, même si l'aspect justification physique doit en souffrir.

\section{Identification du système électro-hydraulique.}

\subsection{INTÉRÊT D’UNE IDENTIFICATION. - Plusieurs cas sont à distinguer :}

- le système existe, l'identification permet à partir de données expérimentales de disposer d'un modèle de commande plus simple et plus efficace que le modèle physique ;

- le système se trouve au stade du projet : il est difficile et dangereux d'extrapoler une identification faite sur une installation de même type ; ceci se justifie par contre davantage pour le modèle physique, qui peut se valider au moins partiellement sur un système voisin ; alors, l'identification des résultats de simulation de ce modèle physique permet la conception et les tests de faisabilité pour la régulation, notamment dans le cas de systèmes à hautes performances, où le matériel de commande, les comportements dynamiques et les réglages souvent «pointus » doivent se déterminer d'avance pour lancer l'opération avec une sécurité suffisante.

Les méthodes utilisées, quel que soit le cas de figure, resteront préférentiellement dans un cadre linéaire, pour les raisons énoncées ci-dessus. Les paragraphes suivants explicitent ces méthodes, et illustrent leurs résultats sur 2 applications; on notera que le passage à un modèle numérique n'est ni un obstacle, ni une obligation.

\subsection{LES MÉTHODES. - L'identification peut fournir :}

- un modèle continu, avec ses repères physiques, susceptible de concorder avec les équations descriptives du paragraphe 1 ;

- un modèle numérique, directement utilisable par le calculateur; à noter qu'un modèle continu se transforme aisément en modèle numérique.

La différence entre ces 2 types d'identification se traduit au niveau méthodologique : dans le cas du modèle continu, on privilégie la ressemblance réponse modèle/réponse réelle ; dans le cas du modèle numérique, d'autres éléments interviennent (filtrage maximum des bruits, par exemple).

Les études effectuées ont mis en œuvre les 2 procédures. Ceci se justifie, dans les applications à hautes performances, par une raison technique : la pression différentielle $\Delta p$ entre les chambres du vérin, qui évolue très vite, peut faire l'objet d'un bouclage interne, à temps de réponse très bref, mais où les autres exigences du cahier des charges sont faibles ; une régulation analogique suffira. La boucle de positionnement sera, elle, toujours numérique, pour offrir le maximum de possibilités de correction.

2.2.1 Identification analogique. - Le signal le plus simple à appliquer à la servovalve est l'échelon; la mesure de $\Delta p$ pose quelques problèmes pratiques (l'actionneur doit être bloqué, d'où stricte limitation de l'amplitude du signal). La procédure se déroule en 2 temps:

- initialisation par le logiciel IDENT [2] qui fournit une série de modèles par des méthodes simples ; 
— amélioration par une méthode du modèle, suivant le schéma ci-dessous.

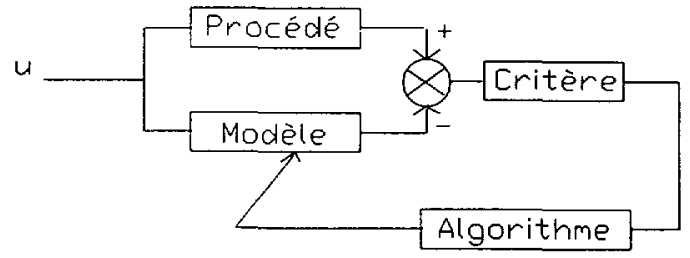

Fig. 4. - Principe de la méthode du modèle.

[Principle of the « model method ».]

2.2.2 Identification par modèle numérique. - Elle a été utilisée pour l'identification du comportement en vitesse de l'actionneur (ce qui est plus simple en simulation, la position s'en déduisant par une simple intégration); lors des essais réels, c'est la position qui est mesurée.

L'identification numérique est basée sur la méthode des « moindres carrés » dont nous rappelons ci-dessous les caractéristiques.

L'équation de récurrence d'un système linéaire d'ordre $n$, en négligeant le retard éventuel qui ne se traduit que par un décalage d'indices, s'écrit, au $(k+1)$-ième échantillonnage, soit à $k T_{\mathrm{e}}$

$$
s_{k}+a_{1} s_{k-1}+\cdot \quad+a_{n} s_{k-n}=b_{0} u_{k}+b_{1} u_{k-1}+\cdot+b_{n} u_{k-n}
$$

en notant $x_{k}$ la valeur de la grandeur $x$ à $k T_{\mathrm{e}}$.

La méthode des «moindres carrés » va admettre que cette relation est vérifiée par les sorties $s_{k}, s_{\ell-1}$, du système réel en tenant compte d'une erreur $\varepsilon_{k}$; les coefficients $a_{\imath}$ et $b_{j}$ du meilleur modèle seront alors ceux qui minimisent le critère $J=\sum_{k} \varepsilon_{k}^{2}$ sur un ensemble d'échantillons, donc de mesures, recouvrant l'«horizon d'observation » du procédé. En mettant les récurrences sous forme matricielle, on a :

$$
S=C \theta+\varepsilon,
$$

où

$$
\begin{aligned}
& C=\left|\begin{array}{lrl}
-s_{n}-s_{n-1} \cdot & -s_{1} u_{n+1} u_{n} \cdot & u_{1} \\
-s_{n+1} & & u_{2} \\
\cdot-s_{N-1}-s_{N-2} \cdot & -s_{N-n-1} & u_{N-n-1}
\end{array}\right| \\
& S=\left|\begin{array}{l}
s_{n+1} \\
. . \\
s_{N}
\end{array}\right| \quad \varepsilon=\left|\begin{array}{l}
\varepsilon_{n+1} \\
. . \\
\varepsilon_{N}
\end{array}\right| \quad \theta=\left|\begin{array}{l}
a_{1} \\
. \\
a_{n} \\
b_{0} \\
. \ddot{b_{n}}
\end{array}\right|
\end{aligned}
$$

Alors le modèle optimal, celui qui minimise $J$ sur $\left[(n+1) T_{\mathrm{e}}, N T_{\mathrm{e}}\right]$, a pour paramètres $\theta_{\text {opt }}=\left(C^{\mathrm{T}} C\right)^{-1} C^{\mathrm{T}} S$. 
En pratique, on préfère éviter l'inversion d'une matrice carrée de dimension $2 n+1$ et on transforme la relation précédente en une formulation récurrente qui permet de déduire le vecteur des paramètres $\theta$ à l'instant $(k+1) T_{\mathrm{e}}$ de $\theta$ à $k T_{\mathrm{e}}$. Cette méthode a été mise en œuvre à partir du progiciel PIM mis au point par I. D. Landau et son équipe, ainsi que du progiciel MATLAB. Le signal d'excitation, conformément aux recommandations des concepteurs de ces logiciels, est une séquence binaire pseudo-aléatoire (SBPA) de faible amplitude. Cette démarche justifie un modèle linéarisé autour d'un point de fonctionnement, mais pas son usage pour toute la gamme des vitesses possibles ; plusieurs essais à divers points de fonctionnement seront nécessaires.

Dans tous les cas, il faut résoudre les problèmes suivants :

\section{- Choix de la période d'échantillonnage $T_{\mathrm{e}}$.}

Pour tirer parti d'un traitement numérique, et compte tenu de la dynamique d'évolution du processus, $T_{\mathrm{e}}$ doit être «grand »; mais il faut en même temps respecter le théorème de Shannon qui impose un maximum égal à la moitié de la période du signal transmis le plus rapide et assurer une reconstitution convenable du signal mesuré. Une valeur telle que $T_{\mathrm{M}}=8 T_{\mathrm{e}}$, où $T_{\mathrm{M}}$ désigne le temps de montée de 10 à $90 \%$ de la réponse finale lors de l'application d'un échelon, a été retenue.

- Choix du type de traitement.

Les données sont centrées et filtrées pour assurer une meilleure représentativité et obtenir une relation significative entre les variations $\Delta v$ de la vitesse et $\Delta u$ du signal de commande appliqué à la servovalve.

\section{- Choix de la structure du modèle.}

La structure classique suivante a été adoptée (les modèles numériques étant exprimés à l'aide de la transformation linéaire « en $z »$ ).

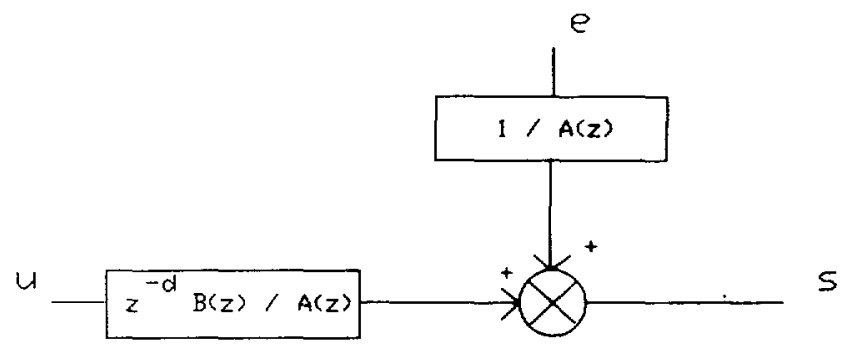

Fig. 5. - Structure des modèles numériques.

[Structure of the numerical models.]

\section{- Choix de la variante méthodologique.}

Nous avons retenu les «moindres carrés généralisés » qui fournissent, en principe, des résultats non biaisés.

\subsection{LES RÉSULTATS. - L'identification a été menée :}

- sur le comportement, simulé numériquement par un modèle physique complet, d'un système de catapultage (moteur hydraulique commandé par une servovalve débit à 2 étages alimentée sous 210 bars),

— sur l'asservissement réel par vérin et servovalve 70 bars d'une charge réglable. 
2.3.1 Identification par modèle analogique. - Pour une réponse indicielle définie par quelque 50 mesures, la coïncidence se fait à mieux que $1 \%$ de la pleine échelle avec une réponse de modèle du $3^{\mathrm{e}}$ ordre (servovalve 210 bars) ou du $2^{\mathrm{e}}$ ordre (servovalve 70 bars), la différence traduisant principalement l'influence de la compressibilité de l'huile.

Ceci corrobore les résultats d'études menées notamment par réduction de modèles physiques, ou par analyse fréquentielle.

2.3.2 Identification par modèle numérique. - Avec une boucle de pression sur la catapulte (boucle construite à partir des résultats de l'identification analogique), les résultats sont bons, et 2 à 3 modèles permettent de «couvrir » le champ des vitesses. On aura donc un modèle à paramètres « glissants ».

Sans boucle de pression, les résultats sont tout à la fois :

- de moindre qualité quant au respect des critères de performance ;

- de moindre robustesse (les identifications à différents points de fonctionnement fournissant des modèles d'ordre différent, à faible plage de validité).

On retrouve là une justification indirecte de la structure de commande multi-boucles décrite au paragraphe 3 .

\section{Structure de la commande.}

Comme pour un moteur à courant continu, la structure de commande va comporter 3 boucles:

- une boucle réglant $\Delta p$ (bouclage «énergétique » analogique)

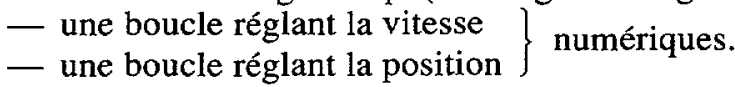

3.1 LA BOUCle DE PRESSION. - Vu sa structure (procédé identifié + capteur de pression, assimilable à un système du $1^{\mathrm{er}}$ ordre, si l'identification s'est faite sur un système simulé), cette boucle aura pour effet :

- de compenser le rôle déstabilisateur du capteur de pression ;

- d'améliorer dans tous les cas la stabilité de la boucle.

Un correcteur à avance de phase, réalisable analogiquement, assurera cet objectif.

3.2 BOUCLE NUMÉRIQUE DE VITESSE. - Le correcteur, mis sous forme de structure $R-S-T$ (3 blocs de transmittance $R(z), S(z)$ et $T(z)$, Fig. 6) se détermine aisément en imposant les pôles de la boucle. Parmi les nombreuses méthodes possibles, celles offertes par le logiciel PC-REG, notamment, se sont avérées bien adaptées aux modèles identifiés. On obtient ainsi un comportement de $2^{\text {nd }}$ ordre prédéfini, tant en asservissement qu'en régulation.

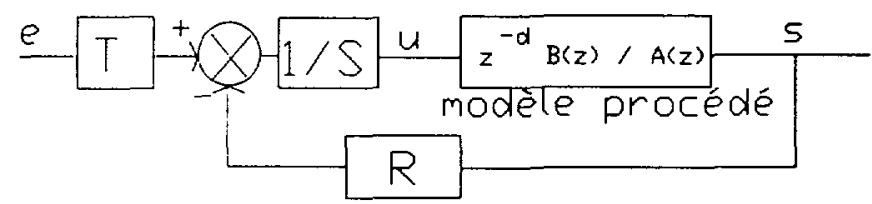

Fig. 6. - Boucle numérique à correcteur $R-S-T$.

[Numerical loop based upon $R-S-T$ controller.] 
3.3 BoUClE NUMÉRIQUe DE POSITION. - La procédure ci-dessus définie s'applique également ici, avec la particularité suivante : les performances souhaitées varient avec le type de fonctionnement, et ce au cours d'un même essai. La simulation aidera à résoudre cette difficulté.

Les correcteurs obtenus, après transformation en équations de récurrence, s'implantent facilement sur tout calculateur.

3.4 Simulation et validation. - Compte tenu des éléments précités (modèles variables, performances demandées elles-mêmes variables), la structure de commande devra comporter une logique de commutation, heureusement aisément modélisable par GRAFCET ou réseau de Petri. Mais une structure aussi complexe, qui jusqu'ici n'a été représentée que par morceaux, exige une simulation globale, qui permettra l'affinage du réglage et constituera la première étape de la validation de la procédure.

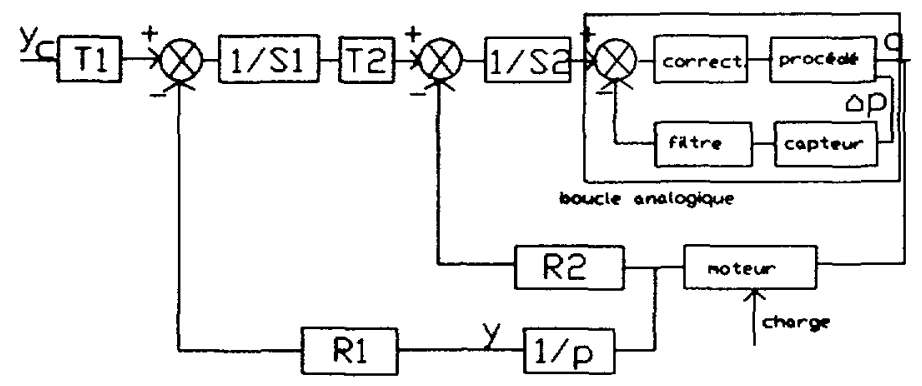

Fig. 7. - Structure de commande à 3 boucles.

[3 loops control structure.]

Cette simulation (cf. courbes de la Fig. 8) montre que pour la boucle de position, malgré un changement de charge lors du mouvement de retour, cas rencontré dans de nombreuses applications, une simple commutation du gain du correcteur suffit à assurer des performances convenables; elle permet aussi de s'assurer de l'absence de saturation des signaux de commande pour un point de fonctionnement «moyen », donc du respect de l'hypothèse de linéarité.

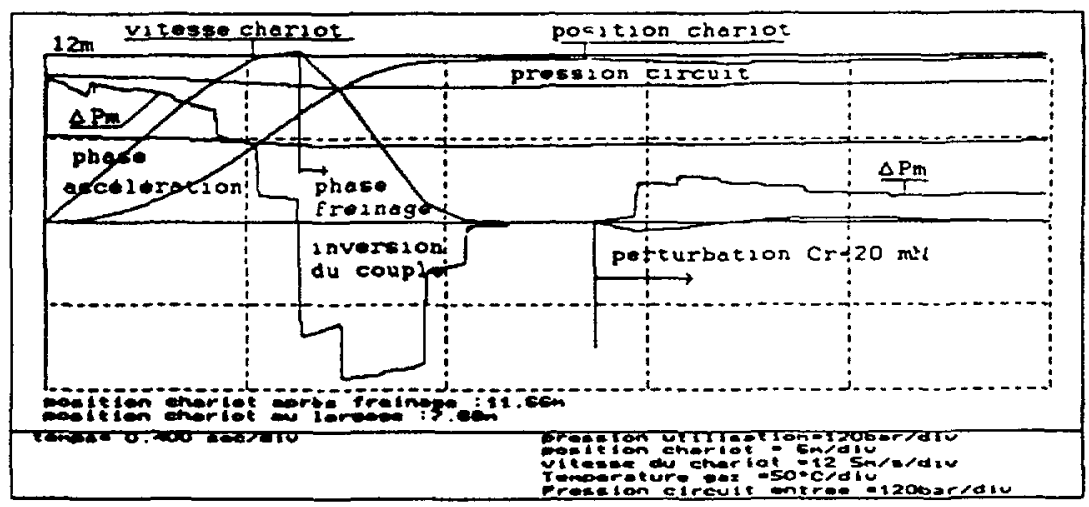

Fig. 8. - Quelques résultats.

[Some results.] 
La validation, dans le cas d'un projet potentiel, s'appuie sur d'autres éléments :

- test du modèle physique; puis de ses dérivés les modèles de commande, obtenus grâce aux méthodes du paragraphe 2, sur un ensemble réel de même nature ;

- vérification de la cohérence physique du fonctionnement simulé par analyse de grandeurs intermédiaires : ainsi la pression, pendant le mouvement de retour d'un dispositif de catapultage, doit être fournie par l'accumulateur; il faut que les valeurs atteintes soient compatibles avec les possibilités de ce dernier;

- faisabilité de la commande :

* l'ajout de sécurités de type logique ne soulève pas de difficulté ;

* la boucle de pression a été rendue analogique, avec des correcteurs à temps de réponse très bref $(<1 \mathrm{~ms})$;

* les calculs des boucles numériques doivent s'effectuer en un temps $<T_{\mathrm{e}}$. Les essais partiels faits sur un PC à processeur 80386 autour d'un programme écrit en langage C montrent que ce résultat est accessible.

\section{Conclusion.}

L'utilisation des techniques numériques de commande autorise :

- la simulation d'un modèle global de fonctionnement, avec ses équations différentielles non linéaires ;

- la détermination par des méthodes d'identification de modèles de commande, numériques ou analogiques suivant les besoins au niveau connaissance aussi bien qu'exécution; l'ensemble de ces modèles constituant ici une approximation, linéaire par morceaux, du modèle précédent;

- l'étude de la commande dans des cas qui dépassent le simple changement de consigne, en $\mathrm{y}$ incorporant notamment les commutations.

Assorties d'une vérification de la cohérence physique et d'une validation, au moins partielle, par des essais réels, ces techniques constituent le fondement d'une étude approfondie de systèmes où l'expérimentation réelle est coûteuse, voire risquée. Aussi sur un projet à niveau d'exigence élevé (catapulte), elles ont justifié l'utilisation de 3 boucles, souvent imposée a priori, et permis de déterminer, pour chacune de ces boucles, des correcteurs simples. Cette concordance avec des constatations pratiques, mais accompagnée ici d'une validation numérique beaucoup plus poussée, prouve bien tout leur intérêt.

\section{Bibliographie}

[1] Bertrand M., Elaboration de modèles de commande à partir de classes de référence, I.M.A.C.S./I.F.A.C. Symposium on Modelling and Simulation (Lille, 1986).

[2] Bertrand M., Note sur le logiciel IDENT, E.N.S.A.M. (Centre de Lille, 1991).

[3] FAISANDIER J. et al., Hydraulique et Informatique (Promotion Presse Internationale, Paris, 1991).

[4] FAYET G., Modélisation d'un système hydraulique, dans Energie Fluide, 8 (décembre 1988).

[5] Hautier J. P., Les organes des systèmes asservis, C.N.A.M. (Lille, 1987).

[6] Houriez S. et Choffardet P., Modélisation et asservissement d'un système hydraulique, Projet de fin d'études, E.N.S.A.M. (Paris, 1991). 
[7] Landau I. D., Identification et commande des systèmes (Hermès, Paris, 1988).

[8] LuUng L., System Identification, Theory for the User (Prentice Hall, Englewood Cliffs, N. J., 1987).

[9] Poulain J., Modélisation d'un système hydraulique. Application au positionnement rapide d'un mobile. Mémoire C.N.A.M. (Lille, 1991).

[10] Sikora M., Hydraulic Control System Analysis Using Network Simulation, Control Engineering (septembre 1991). 